\title{
An energy-efficient opportunity cooperative MIMO transmission scheme in wireless sensor networks
}

\author{
Donglai Hao ${ }^{a}$, Lin Zhou and Zhijie Mao \\ Department of Information transmission, Xi'an Communication Institute, Xi'an,710106, China \\ aemail:hao_dl@126.com
}

\begin{abstract}
Key Words: Wireless Sensor Networks; Multiple-Input Multiple-Output; opportunistic cooperative transmission
\end{abstract}

\begin{abstract}
For only one node assisting the cluster head node to send information in wireless sensor network cooperative transmission, an opportunity cooperative MIMO transmission scheme is proposed based on gradient algorithm. The results of analysis and simulation show that the cooperative transmission schemes based on one- bit feedback can obtain high energy-efficiency while the opportunity cooperative MIMO transmission can obtain higher energy-efficient than it. Then we propose a cooperative MIMO scheme based on cooperative node updating to improve the energy efficiency of cooperative transmission when some nodes can cooperate with cluster head node on transmission. We analyze the total energy consumption of cooperative transmission and the proportion of energy consumption of each node. The results of analysis and simulation show that the proposed scheme can effectively reduce the total energy consumption and balance the energy consumption.
\end{abstract}

\section{Introduction}

Low power consumption is the main characteristics of the wireless sensor network maximizing the lifetime of network is the primary consideration factors in the design of wireless sensor network, especially, the cluster head node and collaborative nodes of power without effective allocation, and in selecting cooperative nodes, not considering the node residual energy is considered cluster nodes and cluster head node between the channel quality, and do not consider the cluster remote node and the number according to the fusion center between the channel condition. From the literature [1], we can know that the transmission power of the transmitter can be adjusted to improve the BER performance such as Alamouti codes under the total power constraint. To adjust the transmission power of the transmitter, the channel state information should be feed back to the transmitter. In the literature [2], it is pointed out that the performance of MIMO system can be improved by using full channel state information, but it is not suitable to be applied to wireless sensor networks with limited energy. In order to compromise the complexity and performance, D J Love in the literature [3] that the use of limited feedback can make the MIMO system to obtain a higher transmission performance. But it is difficult to get all the channel information, especially in the limited energy wireless sensor networks. This paper attempts an opportunistic cooperative mode, to reduce the 
energy consumption of wireless sensor in MIMO transmission.

\section{System model}

The system communication model is shown in figure 1. Each cluster head node $(\mathrm{CH})$ to collect and fusion data from the common nodes $(\mathrm{CN})$, the data was sent to the DFC using time division multiple access (TDMA) algorithm [4]. After the cluster is formatted, the collected data is transmitted to the $\mathrm{CH}$ by $\mathrm{CN}$, and $\mathrm{CH}$ using received signal strength indicator to find cooperative nodes nearest to meet the energy requirements, and cooperative multiple antenna is also formed, cooperation cluster head nodes $(\mathrm{CCH})$ of the transmitted signal code, and then sent to the DFC. In this paper, we mainly focus on the communication between $\mathrm{CH}$ and $\mathrm{DFC}$, and ignore the energy consumption of $\mathrm{CN}$ to send data to $\mathrm{CH}$. In addition, as the energy of DFC is available, the energy consumed by the feedback is negligible. The energy consumption of the whole communication process is mainly focused on the two stages of local communication $(\mathrm{CH}$ and $\mathrm{CCH})$ and long distance communication ( $\mathrm{CCH}$ and $\mathrm{DFC})$.

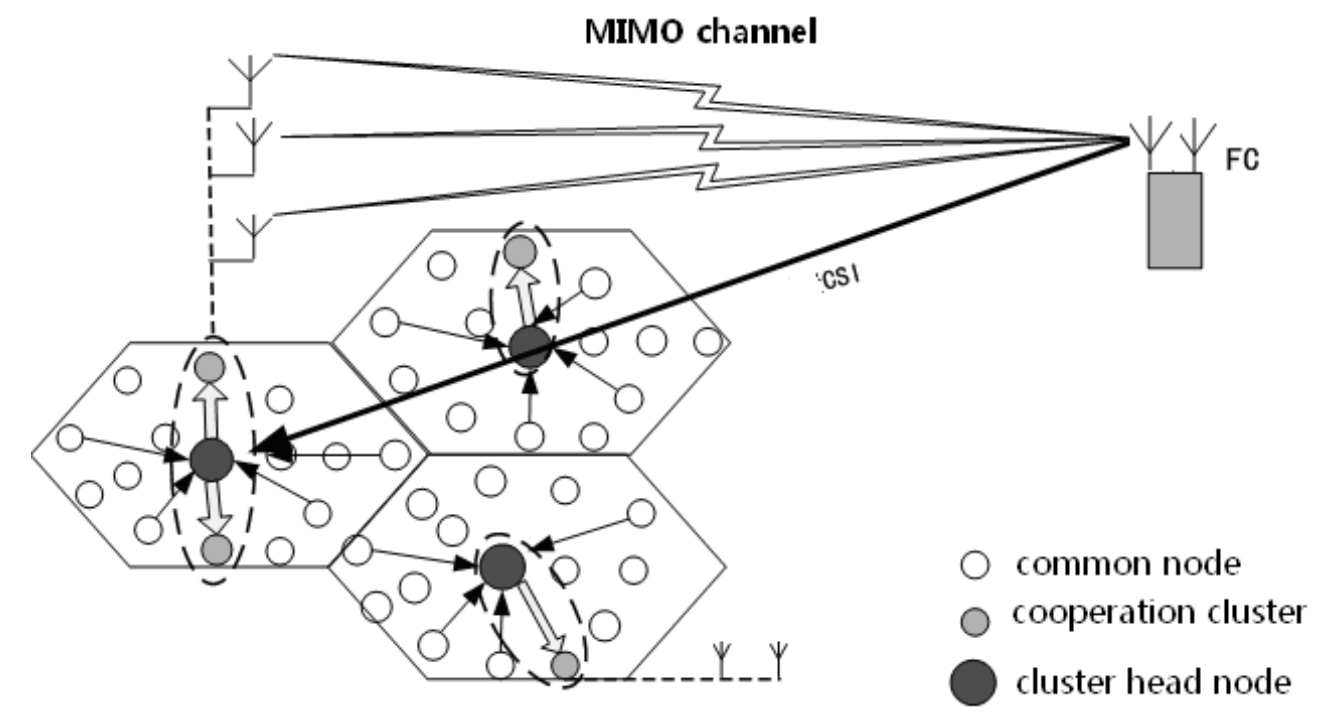

Figure1 cooperative MIMO system with limited feedback

\section{Opportunity cooperative transmission scheme}

When the transmitter does not know the channel state information, signal is transmitted by equal power. when the transmitter obtain the bit feedback information, $\mathrm{CH}$ and $\mathrm{CCH}$ using gradient algorithm to adjust their transmission power. When the feedback bits $b f=1, \mathrm{CH}$ power weight increase $\Delta w$ and decrease $\Delta w$ in $\mathrm{CCH}$, when $b f=0$, power weight decrease $\Delta w$ in $\mathrm{CH}$ and increase $\Delta w$ in $\mathrm{CCH}$, which $\Delta w$ can be determined according to different channel environments. According to the diagonal weight mechanism proposed by [4], At the $n$ moment the weight matrix $\mathbf{w}_{n}$ can be expressed as: 


$$
\mathbf{w}_{n}= \begin{cases}\operatorname{diag}\left\{\sqrt{w_{n-1}+\Delta w}, \sqrt{w_{n-1}-\Delta w}\right\}, & \text { bf }=1 \\ \operatorname{diag}\left\{\sqrt{w_{n-1}-\Delta w}, \sqrt{w_{n-1}+\Delta w}\right\}, & \text { bf }=0\end{cases}
$$

Where $0 \leq \Delta w \leq 0.5,0 \leq w_{n} \leq 1$ When the $w_{n}$ increase to 1 , it will no longer increase; and it will no longer be reduced when $w_{n}$ to 0 .

Opportunistic cooperative transmission scheme is an improved gradient algorithm, The corresponding weight matrix can be expressed by the formula (2)

$$
\mathbf{w}_{n}= \begin{cases}\operatorname{diag}\{1,0\}, & b f=1 \\ \operatorname{diag}\{0,1\}, & \text { bf }=0\end{cases}
$$

Assuming $\xi=\max \left(\left|h_{1}\right|^{2},\left|h_{2}\right|^{2}\right)$, using opportunistic cooperative transmission scheme, the probability density function $\xi$ can be expressed as

$$
f(\xi)=2(\exp (-\xi)-\exp (-2 \xi)), w_{n}=0,1, \xi \geq 0
$$

Similarly, the bit error rate of opportunistic cooperative transmission scheme can be expressed as

$$
\bar{\delta}_{\text {opp }, \mathrm{b}}=\mathrm{E}_{\mathbf{H}}\left\{\boldsymbol{\delta}_{\mathrm{b}}(\mathbf{H})\right\} \leq\left(\frac{4\left(1-M^{-1 / 2}\right)}{b}\right)\left(\frac{3 b E_{\mathrm{b}}}{2(M-1) N_{0}}\right)^{-2}
$$

The total energy consumption of opportunistic cooperative communication divided into the following two parts: a frame data transmission energy consumption and opportunistic communication energy consumption in cooperative MIMO scheme. Because the former method is the same as the energy consumption of the cooperative MISO scheme described in literature [5], it will not be introduced. For the same distribution between $h_{1}(n)$ and $h_{2}(n)$, the probability of $\left|h_{1}\right|^{2} \geq\left|h_{2}\right|^{2}$ and $\left|h_{1}\right|^{2}<\left|h_{2}\right|^{2}$ are both 0.5 . So the total energy consumption is

$$
E_{\text {opp-total }}=0.5 E_{\text {direct }}+0.5 E_{\text {forward }}+N_{\text {frame }} E_{\text {Stb,total }}
$$

Where $E_{\text {Stb,total }}$ is bit energy consumption of cooperative MIMO scheme in the literature [5], $E_{\text {direct }}$ is the energy consumption which data transmission directly from $\mathrm{CH}$ to the DFC. From the above analysis, we can obtain

$$
E_{\text {direct }}=\left(N_{\mathrm{b}}-N_{\text {frame }}\right)\left((1+\alpha) \bar{E}_{\text {opp }, \mathrm{b}} \frac{(4 \pi)^{2} d^{\mathrm{K}}}{G_{\mathrm{t}} G_{\mathrm{r}} \lambda^{2}} M_{\mathrm{l}} N_{\mathrm{f}}+\frac{P_{\text {c-long }}}{b B}\right)
$$

where $\bar{E}_{\text {opp,b }}$ is the required bit energy when adopting opportunistic cooperative communication scheme with a required receiver BER, by the formula (6) can be derived Chernoff upper bound as

$$
\begin{aligned}
& \bar{E}_{\text {opp }, \mathrm{b}-\mathrm{bound}} \approx\left(4\left(2^{b}-1\right) / 3 b\right) N_{0}\left(4\left(1-2^{-b / 2}\right) / b \delta_{\mathrm{b}}\right)^{1 / 2} \\
E_{\text {forward }}= & \left(N_{\mathrm{b}}-N_{\text {frame }}\right)\left(E_{\mathrm{b}-\text { local }} / N_{\mathrm{b}}+P_{\text {c-local }} / b B\right)+E_{\text {direct }}
\end{aligned}
$$

where $E_{\text {forward }}$ is the energy consumption for transmitting data from $\mathrm{CH}$ to $\mathrm{DFC}$ by $\mathrm{CCH}$. 


\section{Simulation results and analysis}

This section gives the performance comparison of two cooperative transmission schemes based on 1 bit feedback and the literature [5]. Scheme 1 in this section below and the figure 1 shows in informed one bit feedback information under the gradient algorithm of cooperative transmission scheme, scheme 2 show in informed one bit feedback information under the opportunistic cooperative transmission scheme. Simulation results is based on table 1 simulation parameters.

Table 1 simulation parameters

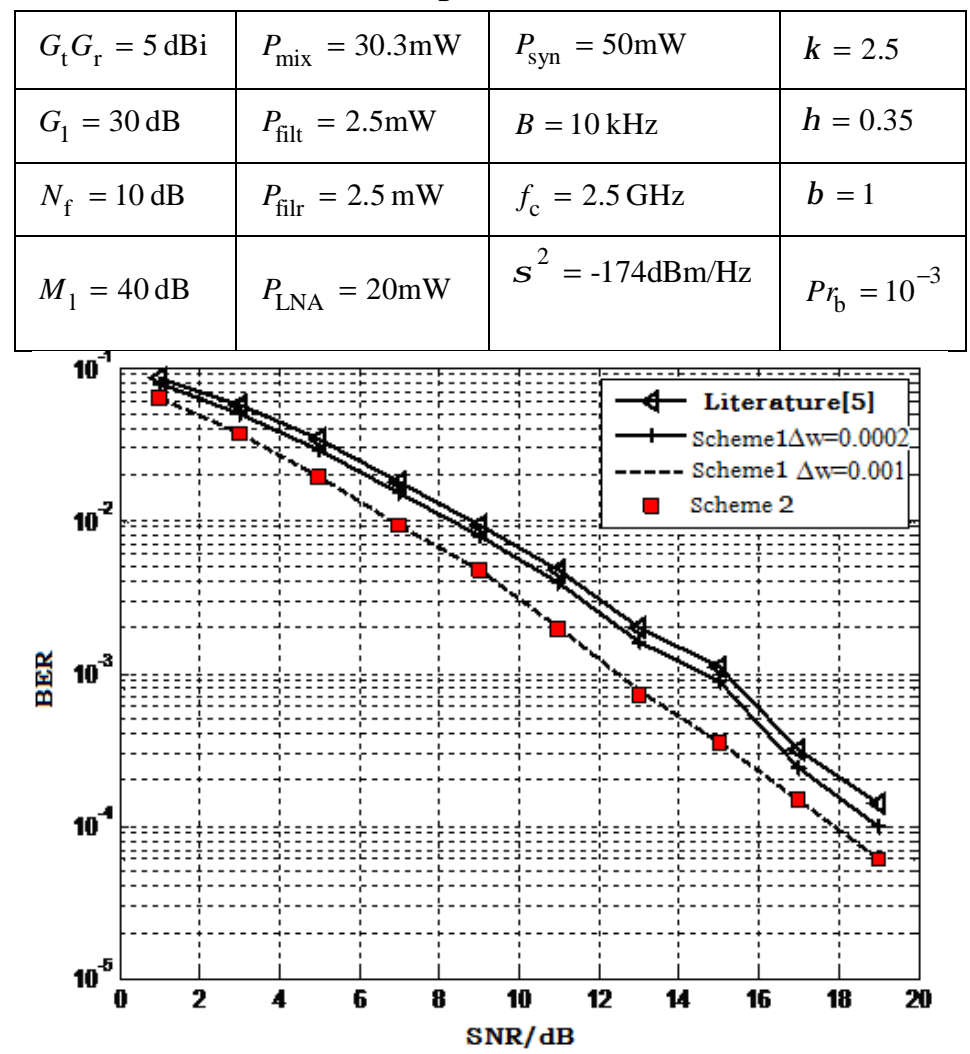

Figure 2 BER vs SNR

We can see from figure 2, under the same signal-to-noise ratio conditions, scheme 1 and 2 are better than the BER performance of the scheme in [5], in other words, scheme 1 and 2 requires lower SNR at the same bit error rate, In addition, it is also known that when the step size of the gradient algorithm is smaller, the performance of the scheme 1 is not improved compared with that of the literature [5], when the step size is larger, the performance of the scheme 1 is close to the performance of the scheme 2 .

\section{Conclusion}

This paper presents a opportunistic cooperative transmission scheme, theoretical analysis and simulation results show that in wireless sensor network, comparing with the gradient algorithm of cooperative transmission scheme, opportunistic cooperative transmission scheme can reduce energy consumption. Although the use of opportunistic cooperative transmission scheme may make the nodes energy consumption not balanced, but the energy consumption of opportunistic cooperative transmission scheme is less than the gradient algorithm. 


\section{Acknowledgement}

This work was financially supported by the Shaanxi Nature Science Foundation (2015JM6325 and 2014JM26101).

\section{References}

[1] Xue Y, Gershman A B. Alamouti-type wireless communication systems with one-bit feedback [J]. IEEE Sensor Array and Multichannel Signal Processing Workshop, 2004, 7:143-147.

[2] Goldsmith A, Jafar S A, Jindal N, et al. Capacity limits of MIMO channels. IEEE Journal on Selected Areas in Communications, 2003, 21(5):684-702.

[3] Love D J, Heath Jr R W, Santipach W, et al. What is the value of limited feedback for MIMO channels? IEEE Communications Magazine, 2004, 42: 54-59.

[4] Yao Y W, Giannakis G B. Energy-efficient scheduling for wireless sensor networks [J]. IEEE Trans. on Wireless Comm., 2005, 53(8): 1333-1342.

[5] Cui S, Goldsmith A J, Bahai A. Energy-efficiency of MIMO and cooperative MIMO techniques in sensor networks [J]. IEEE Jour. Select. Areas of Communications, 2004, 22(6):1089-1098. 